\title{
Onisciente: um modelo de geração de contexto baseado em RFID e sensores
}

\author{
Felipe da Costa Noguez ${ }^{1}$ \\ Bruno Guilherme Martini ${ }^{1}$ \\ Rodrigo Remor de Oliveira ${ }^{1}$ \\ Cristiano André da Costa ${ }^{1}$ \\ Jorge Luis Victória Barbosa ${ }^{1}$
}

\begin{abstract}
Resumo: O monitoramento de entidades para determinar uma situação é uma aplicação interessante para diversas áreas. Pode-se utilizar dados obtidos de sensores, disponíveis em um ambiente, para descrever o contexto dos elementos presentes. Nesse âmbito, o presente trabalho tem como finalidade apresentar o Onisciente, um modelo que utiliza a informação de um conjunto de sensores que estejam monitorando um mesmo elemento para determinar o seu contexto e obter conhecimento sobre suas condições. Para isso, o Onisciente realiza a identificação dessas entidades, com o auxílio de uma leitora RFID acrescida de alguns sensores. No modelo, um contexto representa uma imagem de momento preciso no tempo, na qual se pode verificar fatos que estejam ocorrendo e, com isso, tomar decisões. Essas decisões envolvem classificar os contextos gerados e, conforme a classificação obtida, informar eventuais situações desfavoráveis às entidades monitoradas. Para determinar um contexto e realizar a classificação do significado que ele representa, são empregadas ontologias, computação sensível ao contexto e modelagem de contexto. Um protótipo foi desenvolvido para avaliar o modelo, mostrando a viabilidade do modelo proposto.
\end{abstract}

Palavras-chave: Computação móvel. Sensibilidade ao contexto. Sensores. Web semântica.

\begin{abstract}
The monitoring of entities to define a situation is an interesting application for various areas. We can use data gathered from sensors, available in the environment, to describe the context of the present elements. In this way, this article proposes Onisciente, a model that uses information obtained from a set of sensors monitoring the same element to determine its context and generate knowledge about its condition. To do that, Onisciente makes the identification of those entities, using an RFID reader with some sensors. In the model, a context represents a snapshot of that precise moment in time, in a way that facts can be detected and decisions can be made. These decisions involve classify the generated contexts, and accordingly inform bad situations to the monitored entities. To determine the context and make the classification of their mening, we employed ontologies, context-aware computing and context modeling. We developed a prototype to evaluate the model, showing the proposed model feasibility.
\end{abstract}

Keywords: Mobile computing. Context awareness. Sensors. Web semantic.

\section{Introdução}

Hoje, o RFID é utilizado em diversos tipos de aplicações e em diversas áreas, que vão desde controle de acesso até monitoramento, como, por exemplo, na cadeia de suprimentos e na área da saúde [1]. Ao utilizar o RFID, é possível determinar a posição de uma entidade dentro de um ambiente, graças a algumas técnicas de localização existentes [2].

Uma entidade é qualquer ser vivo, objeto ou local que possa ser monitorado. Ao se definir a localização de pelo menos duas ou mais entidades, ao mesmo tempo, e em um mesmo ambiente, obtêm-se condições de

\footnotetext{
${ }^{1}$ Programa de Pós-Graduação em Computação Aplicada - Unisinos.

\{fcnoguez@gmail.com, brunogui92@gmail.com, rodrigo.remor@gmail.com, cac@unisinos.br, jbarbosa@unisinos.br\}
}

http://dx.doi.org/10.5335/rbca.2015.4106

Revista Brasileira de Computação Aplicada (ISSN 2176-6649), Passo Fundo, v. 7, n. 1, p. 53-67, abr. 201553 
descrever a ocorrência de uma situação em uma determinada fração de tempo [3]. Essa situação representa um contexto $[4,5]$, que pode ser definido como uma visão com informações de momento.

Alguns modelos de RFID [6] permitem que sejam anexados sensores [1], elevando a quantidade e tipos de dados que podem ser adquiridos, fornecendo uma variedade de informações que, se adicionada a um contexto existente, admite que esse seja complementado, aumentando o grau de precisão e o nível de detalhamento das condições em que as entidades, nele descritas, se encontram.

Pode-se definir contextos a partir da combinação de informações brutas de diversas fontes individuais e variadas [7], as quais podem ser exploradas em diversas áreas. Por exemplo, na saúde [8], contextos resultantes do monitoramento de pacientes por meio de sensores podem elevar o nível de agilidade e precisão na análise de médicos; na cadeia de suprimentos e logística, podem ser obtidas informações sobre as condições de uma mercadoria em qualquer momento da fase de produção, transporte ou armazenamento, além de disponibilizar conhecimento sobre as condições das estradas e outros dados, como tempo de viagem e tráfego.

Com base nisso, este trabalho apresenta um modelo que faça a identificação de entidades por meio do uso de RFID [6, 7] e, também, uma síntese das condições em que as entidades se encontram, por meio de contextos, mediante a coleta de informações adquiridas por diversos tipos de sensores. Esse modelo é chamado Onisciente, pois o seu principal objetivo é permitir ter conhecimento sobre todas as condições de uma entidade. A principal razão para escolha do uso da tecnologia RFID no trabalho advém da popularidade da tecnologia, aliada ao atual baixo custo.

A principal contribuição do Onisciente é a geração de contextos por meio do tratamento e da combinação de dados, a partir de inúmeras e variadas informações, adquiridas por diferentes sensores, pois os trabalhos encontrados na área possuem foco em aplicações específicas e tendem a abordar partes do processo realizado pelo Onisciente, não sendo capazes de fornecer os mesmos resultados. Ele foi constituído de forma que possa ser utilizado em variados tipos de estudos, projetos e diversas áreas.

Na seção 2, são apresentados os trabalhos relacionados em conjunto com definições e conceitos sobre contexto, RFID, sensores e demais assuntos envolvidos neste trabalho. A seção 3 apresenta a descrição do modelo do Onisciente para criação de contexto a partir de dados obtidos pelos sensores e RFID. Na seção 4, procede-se ao detalhamento do processo de implementação do protótipo. Na seção 5 , são descritos os estudos de casos e cenários utilizados para realizar a avaliação do Onisciente. Por último, a seção 6 apresenta as considerações finais.

\section{Trabalhos relacionados}

A literatura cita diversas definições para contexto [9]. Uma das primeiras foi apresentada por Schilit e Theimer [10], que a definiram como sendo "a localização de uso, a coleção de pessoas e objetos próximos, assim como as mudanças sobre esses objetos ao passar do tempo". Uma das mais utilizadas, porém, é a de Dey [4], para quem "contexto é qualquer informação que possa ser utilizada para caracterizar a situação de uma entidade. Uma entidade é uma pessoa, lugar ou objeto que seja considerado relevante para a interação entre um usuário e uma aplicação, incluindo o usuário e a aplicação".

Contexto pode ser composto por informações referentes a ambiente, localização, aspectos sociais e tempo [11], informando, além das condições climáticas, onde uma entidade está localizada, quem está ao redor e o que existe em sua proximidade. Diferentes campos da Ciência da Computação, como processamento de linguagem natural, aprendizagem de máquina, visão computacional, suporte à decisão, recuperação de informação, computação ubíqua e segurança, utilizam contexto para adicionar adaptação e efetividade na tomada de decisões [9]. A computação ubíqua está intimamente relacionada ao contexto, devido à heterogeneidade e à ubiquidade de comunicação desse tipo de ambiente, onde se necessita adaptação em tempo real às situações de uso ou contexto.

Conforme Bouet e Santos [2] e Liu et al. [6], o RFID é um meio de armazenar e recuperar dados mediante a transmissão eletromagnética para um circuito de rádio frequência (RF) compatível, podendo ser utilizado para identificação e localização. De acordo com Want [12], o RFID permite identificação a distância sem a necessidade de uma linha de visão e pode distinguir variados tipos de identificadores em uma mesma região sem a necessidade de assistência humana.

De acordo com Roussos e Kostakos [1], o papel do RFID na sensibilidade ao contexto é como uma ferramenta de suporte para identificar entidades próximas, permitindo ao sistema fornecer serviços baseados em localização. Um exemplo disso é o emprego do RFID em casas inteligentes, onde, ao haver aproximação de um determinado item, o ambiente pode reagir de uma maneira específica. O RFID é efetivo em identificar a presença 
de entidades marcadas, mas não pode fornecer qualquer informação sobre a sua situação. Uma solução para essa condição é incorporar sensores às tags RFID. No entanto, os sensores necessitam de uma fonte de energia e embora tags RFID ativas possam suprir essa necessidade, não é o que ocorre com tags passivas.

$\mathrm{Na}$ literatura, existe uma ampla variedade de publicações que abordam o uso de RFID e contexto para diversas finalidades. Durante as pesquisas realizadas, não foram encontrados trabalhos que fossem diretamente ao encontro da proposta deste modelo, o de gerar modelagem de contexto baseado em informações fornecidas por tags RFID com sensores. Dessa forma, foram selecionados trabalhos que apresentassem relevância para o objetivo proposto, os quais foram divididos em dois grupos. O primeiro grupo apresenta uma relação de trabalhos que tratam informações obtidas por meio de RFID, realizando modelagem de contexto, resultando em informações semânticas. O segundo grupo descreve trabalhos que exploram o uso de sensores em conjunto com RFID para adquirir informações que possam exercer alguma influência sobre entidades que estejam sendo monitoradas.

Os trabalhos relacionados $[13,14,15]$ do grupo 1 são apresentados na Tabela 1, que apresenta uma compilação das características destacadas dos trabalhos apresentados. Nesta tabela, são exibidas informações relacionadas ao tipo de ontologia utilizada, qual o tipo de tecnologia RFID empregada, se a tecnologia RFID utiliza o padrão EPC, quais os tipos de sensores suportados, qual a arquitetura utilizada em suas propostas, o tipo de modelagem de contexto empregada e qual o meio de representação de contexto utilizado.

Tabela 1: Trabalhos que geram contexto a partir de informações obtidas pelo RFID

\begin{tabular}{l|c|c|c}
\hline Trabalho & Sheng et al. [13] & Catarinucci et al. [14] & Zhang, Zheng, Liu [15] \\
\hline \hline Tipo de RFID utilizado & Não especifica & Passiva & Não especifica \\
\hline Padrão EPC & Sim & Não & Não \\
\hline Tipo de sensores & Variados & Variados & SOA especifica \\
\hline Arquitetura & 3 camadas & 3 camadas & Ontologia \\
\hline Modelagem de contexto & $\begin{array}{c}\text { Ontologia/Baseada em } \\
\text { regras }\end{array}$ & $\begin{array}{c}\text { Ontologia/Baseada em } \\
\text { regras/MultiAgente }\end{array}$ & OWL-S \\
\hline Representação de contexto & Tuplas & OWL & \\
\hline $\begin{array}{l}\text { Formato de representação da } \\
\text { Ontologia }\end{array}$ & SOCAM/OWL & & \\
\hline
\end{tabular}

Tabela 2: Trabalhos que utilizam RFID com sensores

\begin{tabular}{l|c|c|c}
\hline Trabalho & Delen et. al [16] & Kang et. al [17] & Escribano et al. [18] \\
\hline \hline Tipo de RFID utilizado & Semipassivo & Passivo/ Ativo & Passivo/ Ativo \\
\hline Padrão EPC & Sim & Sim & Sim \\
\hline Tipo de sensores & Temperatura & Temperatura & Temperatura/ Umidade \\
\hline Arquitetura & 3 camadas & 3 camadas & SOA \\
\hline Uso de perfil & Temperatura & Temperatura & Nãiliza \\
\hline Método de coleta de dados & Periodoc Pooling & Periodoc Pooling & Não \\
\hline Alarmes de monitoramento & Sim & $\begin{array}{c}\text { Registrar valores de } \\
\text { temperatura (até 64 } \\
\text { registros) }\end{array}$ & $\begin{array}{c}\text { Tags ativas armazenam } \\
\text { dados das cargas } \\
\text { Função do armazenamento no } \\
\text { RFID }\end{array}$ \\
\hline
\end{tabular}

Os trabalhos apresentados $[16,17,18]$ no grupo 2 abordam a utilização de sensores com RFID para obter as condições de entidades em determinados momentos. A Tabela 2 compila as características em destaque dos trabalhos deste grupo, acentuando aquelas que mais interessam para o desenvolvimento do Onisciente. Cita-se, nessa tabela, o tipo de RFID empregado, se as tags usam o padrão EPC, os tipos de sensores utilizados nas avaliações dos modelos, a arquitetura empregada no desenvolvimento dos sistemas, se utilizam perfis em algum 
ponto do sistema, qual o método de captura de dados empregados, se há geração de alarmes de monitoramento e qual a função de armazenamento do RFID.

Os três trabalhos apresentados abordam a área de cadeia de suprimentos, devendo as condições dos produtos transportados ser monitoradas para que haja uma melhor qualidade. Por esse motivo, o foco desses trabalhos está apenas na obtenção dos dados, não fazendo menção a posteriores tratamentos.

\section{Modelo Onisciente}

Esta seção apresenta o modelo Onisciente. No decorrer da seção, será apresentada uma visão geral do modelo, seguido dos requisitos envolvidos. Logo em seguida, será descrita a especificação da arquitetura dos elementos que compõem o modelo, junto com alguns diagramas de atividade que demonstram os procedimentos e as visões do modelo.

\subsection{Visão geral}

O Onisciente utiliza dados adquiridos por meio de tags RFID para criar informações contextuais que exponham as condições de entidades que estejam sendo monitoradas. A Figura 1 apresenta uma visão geral do modelo. Usuários poderão cadastrar as entidades que desejam monitorar por meio de uma interface fornecida pelo cliente. Nesse cadastro de entidade, os usuários especificam um perfil, que abrange as características da entidade e onde estarão presentes as limitações às quais ela pode ser exposta, por exemplo, uma carga que possui uma faixa de temperatura máxima e mínima na qual deve ser mantida, ou um paciente em um hospital que não possa ultrapassar um determinado valor na sua taxa de glicose.

Figura 1: Visão geral do modelo

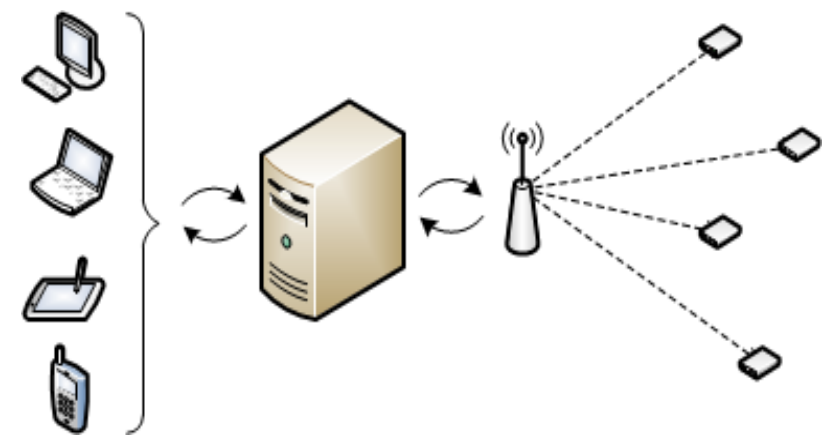

Um recurso oferecido pelo Onisciente é o acompanhamento de entidades por meio de sessões. Uma sessão é um espaço controlado pelo servidor que pertence unicamente ao usuário que a criou, em que ele pode selecionar as entidades que deseja monitorar e por meio dela receber as informações relacionadas a essas entidades. $\mathrm{O}$ modelo fornece aos usuários não apenas informações que indiquem as condições atuais das entidades monitoradas, mas também recursos que disponibilizam a possibilidade de verificar as situações em que as entidades monitoradas foram expostas desde o início do processo de monitoramento.

As informações contextuais geradas proporcionam, no mínimo, a localização da entidade, visto que, existindo uma simples tag RFID [19], é possível determinar a sua localização por meio de técnicas de triangulação ou medição de intensidade de sinal. A qualidade do resultado obtido na geração do contexto dependerá da quantidade de sensores envolvidos no processo; quanto mais diferentes tipos de sensores utilizados e, consequentemente, tipos de dados obtidos, mais completo será o resultado.

Devido à necessidade de utilizar sensores junto às tags RFID, o Onisciente utiliza tags RFID ativas porque elas possuem fonte de energia própria que alimenta os sensores envolvidos, mesmo quando as tags não estão ao alcance de uma leitora. Para essas situações em que não há comunicação, os dados coletados são armazenados na tag para transmissão quando houver contato com uma leitora. Sensores que não estejam conectados às tags RFID também podem ser utilizados, desde que apresentem dados relevantes para a entidade para a qual o contexto é construído, por exemplo, sensores que estejam monitorando as condições de um ambiente específico onde a entidade esteja presente. 
A geração do contexto é realizada e representada por meio de ontologia. Seguindo a tendência identificada ao analisar os trabalhos relacionados, a ontologia em OWL aparenta ser a mais indicada. Possuindo o resultado do contexto criado, as informações obtidas estão disponíveis para os usuários por meio de serviços fornecidos pelo servidor que realiza o processamento dos dados. Esses serviços podem ser consumidos tanto por estações de trabalho quanto por notebooks e outros dispositivos móveis, tais como smartphones e tablets. Um cliente web está disponível para estações de trabalhos tradicionais e notebooks realizarem o monitoramento e poderem efetuar a visualização dos resultados. Já para os dispositivos móveis, um cliente realiza o processo de consulta de informações junto ao servidor para a exibição dos resultados. Ambos os clientes possuem a mesma base e apresentam as mesmas funcionalidades, sendo o diferencial entre eles a maneira como é implementada cada versão. Seu objetivo é aproveitar os recursos oferecidos pelos dispositivos móveis.

Alguns requisitos foram definidos para atender às especificações descritas na visão geral, entre os quais a utilização de uma arquitetura cliente servidor para a utilização de serviços. A utilização de ontologias para a criação e representações de contextos, bem como a permissão da visualização da situação da entidade em qualquer momento em que ela esteve sendo monitorada são aspectos importantes do projeto. O Onisciente também tem capacidade de suportar variados tipos de entidades e fornecer suporte a diversos tipos de dispositivos móveis, como tablets e smartphones.

A quantidade de fontes de dados para a criação dos contextos deve ser indefinida, e o modelo deve permitir o uso de variados tipos de sensores para a aquisição de dados relacionados à entidade monitorada. $\mathrm{O}$ cadastro e a edição de um perfil da entidade a ser monitorada devem ser permitidos pelo modelo. A utilização de tags RFID ativas para o monitoramento de entidades é outro requisito importante para o Onisciente, juntamente com a detecção e a notificação dos usuários sobre situações de risco de uma entidade.

\subsection{Arquitetura}

A arquitetura do Onisciente (Figura 2) foi projetada utilizando o modelo TAM da SAP [20] e é definida como cliente servidor, fornecendo web services para diversos tipos de dispositivos móveis e desktop. O servidor é responsável por adquirir e processar os dados das entidades monitoradas, convertendo-os em informação contextual. O cliente, por sua vez, consome a informação contextual gerada, além de disponibilizar recursos para a interação do usuário com o servidor. Ele está apresentado em duas opções, um site web que pode ser acessado por computadores pessoais, notebooks e dispositivos móveis, e um aplicativo para dispositivos móveis.

Figura 2: Arquitetura Geral do Modelo.

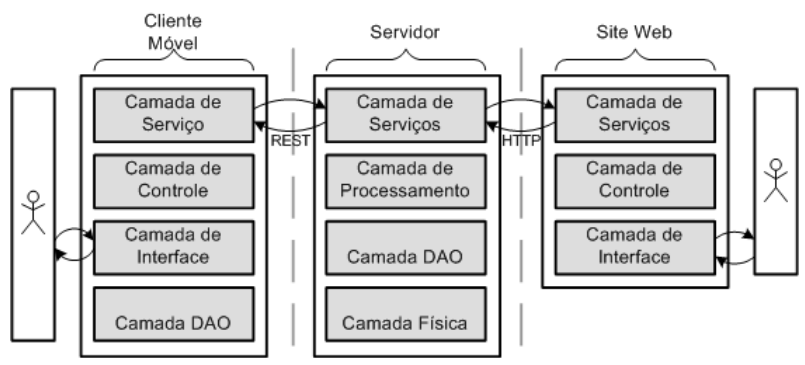

O servidor utiliza uma estrutura em quatro camadas (Figura 3). A camada mais baixa, denominada de Camada Física, é composta pela estrutura de hardware que realiza a aquisição de dados por meio do RFID. Nela estão inseridas as tags RFID ativas e as leitoras, bem como os sensores que estiverem disponíveis.

A segunda camada é composta pelos elementos que convertem dados brutos adquiridos pela primeira camada. Essa camada, denominada de Camada de Processamento, é composta por três módulos que realizam funções distintas. O primeiro módulo é o Gestor de Hardware, que tem como função receber os dados das leitoras RFID e tratá-los de forma que não haja redundâncias nem conflitos de informação, para que possam ser aplicados no segundo módulo. O segundo módulo, Gestor de Ontologia, é abastecido pelo Gestor de Entidades e recebe os dados processados do Gestor de Dados para aplicá-los na Ontologia, resultando em uma informação contextual que será utilizada pelo terceiro módulo. O terceiro módulo é o Gestor de Entidades, e por meio dele o servidor interage com os clientes, recebendo e efetuando as operações de cadastro ou edição de entidades, bem como abastecendo o Gestor de Ontologia para manter a base ontológica atualizada. Outra funcionalidade desse 
módulo é a geração de alarmes quando necessário. Os alarmes apresentam diversos níveis de gravidade e ocorrem quando os limites de um ou mais atributos descritos no perfil de uma entidade são ultrapassados por algum dado existente no contexto. Um alarme pode variar de uma simples notificação de alerta até uma notificação de situação crítica. Um quarto módulo, denominado de Gestor de Sessão, gerencia as requisições de monitoramento de um usuário, mantendo as listas de entidades monitoradas e interage com o Gestor de Entidades para consultar a situação destas entidades, notificando o usuário caso exista algum alarme.

A terceira camada é a Camada DAO. Ela é composta por dois módulos e fornece recursos para que os módulos da Camada de Processamento tenham acesso aos dados armazenados. O primeiro módulo é o DAO Entidades. Ele é responsável por realizar a persistência dos dados relacionados às entidades e sessões criadas pelos usuários na base no banco de dados BD Entidades. O segundo módulo é o DAO Ontologia, o qual realiza persistência de dados no BD Ontologia. Esse módulo é de uso exclusivo do Gestor de Ontologia.

A última camada é a Camada de Serviços, a qual fornece recursos para a comunicação entre os clientes e o servidor. Ela está dividida em dois grupos: o primeiro é constituído de apenas um módulo, nomeado Interface Física $\mathrm{E} / \mathrm{S}$, o qual contém um conjunto de métodos que permite que a camada física interaja com o servidor, realizando o envio de dados; o segundo grupo é composto por diversos módulos que realizam a interação entre o servidor e os clientes do Onisciente por meio do protocolo REST. Esses módulos estão divididos por tipo de controle que eles realizam. O Módulo de Entidade realiza operações relacionadas aos procedimentos de CRUD de entidades, e o Módulo de Sessão, ao controle de sessões e entidades que estão sendo monitoradas.

Figura 3: Estrutura das quatro camadas do servidor.

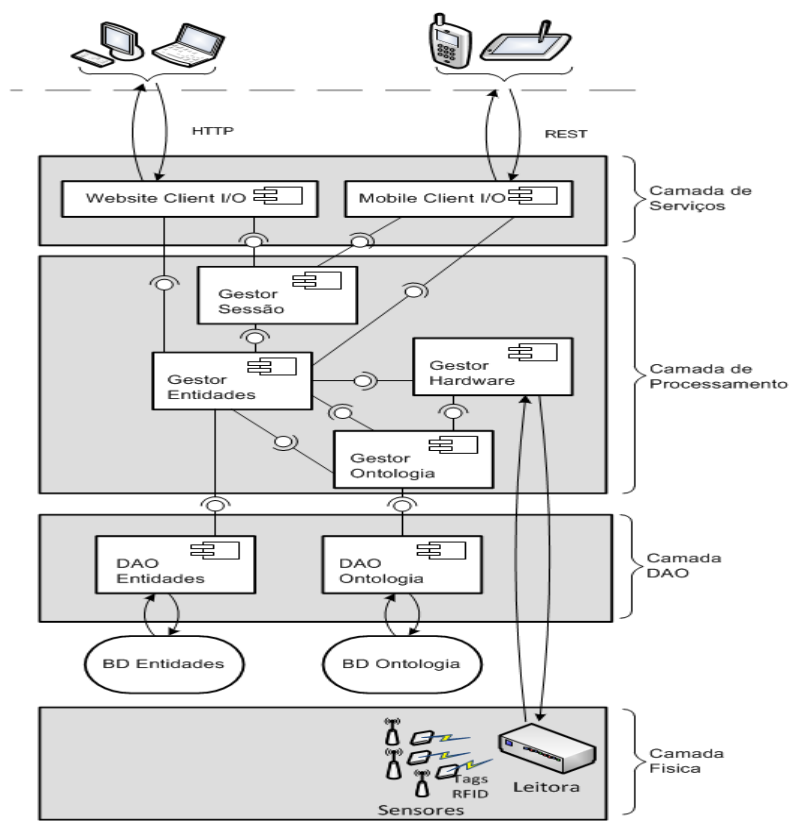

$\mathrm{Na}$ Figura 4a, é possível perceber a existência de ligações entre as diversas classes definidas na ontologia, as quais representam as propriedades das classes e definem as relações entre elas. Ao ser inserida uma pessoa na ontologia, a informação deve informar o lugar onde ela está inserida e, se for o caso, também informar a relação de objetos que essa pessoa possui. Objetos podem estar inseridos ou fazer parte de outros objetos maiores, assim como ser o resultado final da união de diversos objetos menores; além disso, devem estar incluídos em algum lugar e podem pertencer a uma pessoa. Os lugares são uma referência para a definição da localização de objetos e pessoas dentro da ontologia. A classe contextos aparenta estar desconexa das demais classes na Figura 4a, isso porque ela não está expandida e não podemos ver a sua estrutura hierárquica. Cada entidade apresenta um grupo de contextos relacionados, os quais indicam quais são as entidades que estão, de alguma maneira, fora das condições ideais. Objetos são avaliados segundo os seus atributos, pessoas possuem regras predefinidas que determinam algum fator de risco. Por exemplo, é conhecido que a temperatura de uma pessoa deve variar entre $36^{\circ} \mathrm{C}$ e $37^{\circ} \mathrm{C}$ para ser considerada normal, e qualquer valor de temperatura fora desse intervalo é considerado febre. Também é conhecido que alguns intervalos de umidade são prejudiciais para a saúde de uma pessoa. 
Lugares não possuem contextos definidos, porque são os meios como os contextos são avaliados. Um objeto só pode ser avaliado sabendo-se onde ele está inserido; o mesmo vale para uma pessoa. Um detalhe que justifica a aparente desconexão da classe contexto com as demais classes da ontologia, conforme visto na Figura 4a, é o fato de que as subclasses de contexto são classes derivadas das classes de entidades. A Figura $4 \mathrm{~b}$ mostra a estrutura das classes de contexto.

Figura 4: Ontologia proposta pelo Onisciente.

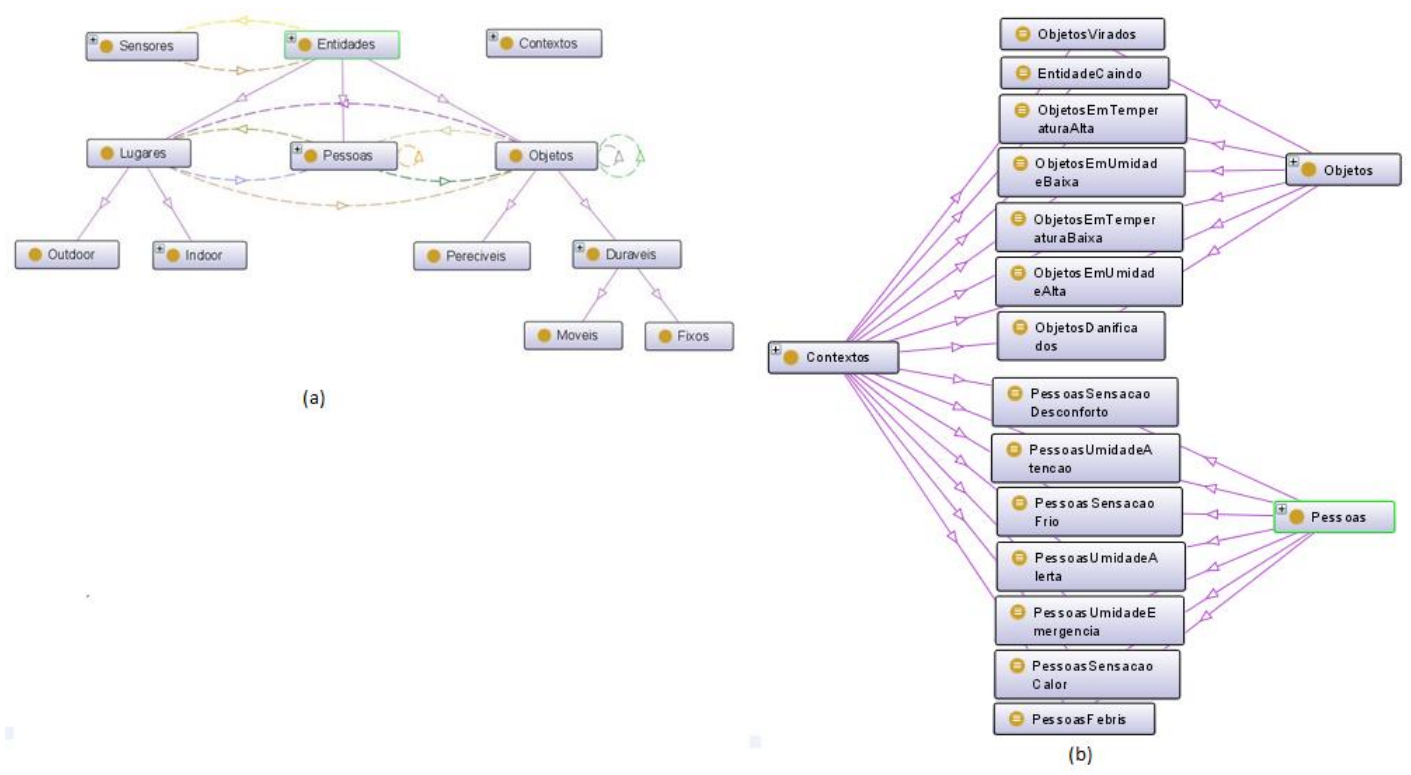

\section{Implementação}

\subsection{Tecnologias utilizadas}

Para o desenvolvimento do servidor e site web do Onisciente, foi utilizada a linguagem C\#, com a ferramenta Visual Studio 2012. O banco de dados foi elaborado em SQL Server 2008 R2 e, para gerenciar a conexão entre o SQL Server e o Visual Studio, foi utilizado o ADO.NET Entity Data Model. Esse recurso cria uma camada de objetos no projeto do Visual Studio que reflete a estrutura modelada no banco de dados e fornece as classes que serão utilizadas na aplicação. Para interagir com essa camada de objetos, foi utilizado o LINQ5. A biblioteca Newtonsoft Json6 foi utilizada para serializar dados para a troca de mensagens com os clientes Android e web. O desenvolvimento do cliente Android foi realizado em Java, utilizando Eclipse com suporte ao ADT (Android Development Tool). A biblioteca Gson7 (Google Json) foi utilizada para auxiliar na troca de mensagens com o servidor. Essa biblioteca é equivalente ao JSON utilizado no Visual Studio.

Figura 5: Equipamentos utilizados para obter dados para o Onisciente.

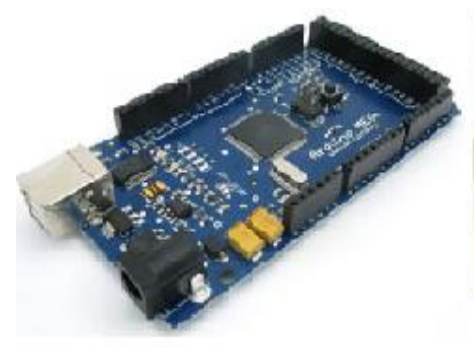

(a)

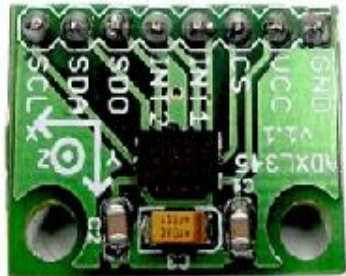

(b)

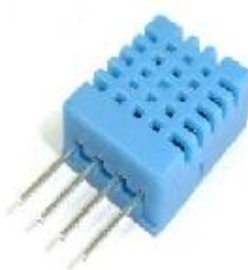

(c)

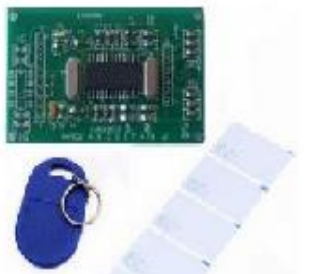

(d) 
Para a coleta de informações, foram utilizados equipamentos disponíveis no MobiLab ${ }^{3}$ (Laboratório de Computação Móvel e Ubíqua da Unisinos). O principal dispositivo empregado consistiu de um hardware composto por um Arduino Mega 2560 (Figura 5a) acoplado com uma leitora RFID de 13,56 MHz (Figura 5d). Essa leitora pode realizar captura de informações a uma distância de até seis centímetros de uma tag, suporta ISO14443A/MIFARE e identifica automaticamente a presença de tags sem a necessidade de comandos. O dispositivo criado para o protótipo também é composto de um sensor DHT-11 (Figura 5c), para obter informações de temperatura e umidade, e de um acelerômetro de três eixos ADXL345 (Figura 5b), que é capaz de identificar o movimento ou a inatividade de entidades, assim como medir a força do movimento ou detectar colisões. O dispositivo resultante da combinação desses elementos pode ser visualizado na Figura 6 . Um telefone celular executando o sistema operacional Android foi utilizado como intermediário para realizar o envio dos dados obtidos para o servidor. O telefone se comunica com o Arduino por meio de uma conexão Bluetooth. Tal procedimento se tornou necessário porque o Arduino não possuía meios de enviar os dados diretamente para o servidor, por falta de um módulo GPRS.

Figura 6: Hardware utilizado no Onisciente.

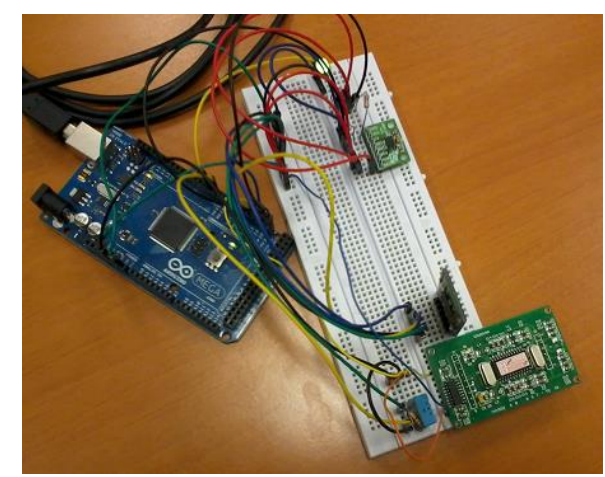

\subsection{Implementação do servidor}

O servidor foi desenvolvido conforme a especificação apresentada no modelo. Foram implementadas as quatro camadas propostas, sendo uma composta por elementos de hardware e o restante por software. O protótipo foi desenvolvido utilizando uma arquitetura orientada a serviços. O foco foi gerar contextos com base na análise de dados de sensores, explicitamente, envolvendo temperatura, umidade e gravidade.

Como resultado da implementação, 21 classes foram criadas para atender o objetivo proposto para o Onisciente. A Figura 7 apresenta o diagrama de classes, no qual elas estão distribuídas da seguinte forma: três na Camada de Serviços para interação com hardware, web sites e dispositivos móveis; quatro na Camada de Processamento para realizar a gestão de dados conforme o tipo; quatorze na Camada DAO, sendo cinco para gerir as entidades mapeadas pelo banco de dados e as dez restantes para as entidades mapeadas propriamente ditas.

\subsection{Implementação dos clientes}

As duas opções de clientes, web e Android, são basicamente terminais de uso, em que o usuário solicita uma ação, essa ação gera uma requisição para o servidor, e este a processa gerando um retorno que é apresentado ao usuário. Não há a realização de processamento no lado dos clientes, a não ser por algumas validações nos dados para garantir que não se consuma largura de banda, enviando mensagens desnecessárias entre o cliente e o servidor. Ambos os clientes utilizam os mesmos serviços disponíveis pelo servidor e ficam responsáveis por tratar a informação que vem serializada em JSON conforme a sua necessidade.

Ambos os clientes desenvolvidos apresentam o mesmo comportamento para a requisição das informações de contexto. No cliente Android, um timer realiza a chamada do serviço do servidor em um período de tempo predeterminado. Já no cliente web, uma função AJAX é responsável por realizar essa chamada. Esse ciclo é

${ }^{3}$ Homepage do MobiLab: http://www.unisinos.br/lab/mobilab/ 
quebrado apenas se houver uma mudança na lista de entidades a serem monitoradas, tanto ao adicionar quanto ao remover entidades da lista. Quando isso ocorre, a requisição é feita de forma automática e o timer é reiniciado.

O cliente Android foi desenvolvido seguindo as especificações definidas pelo modelo apresentado na sessão anterior. A única exceção foi o processo de armazenamento local, que permitiria a um usuário consultar os contextos passados de entidades que tivessem sido monitoradas. Com isso, a arquitetura da aplicação reduziu o número de camadas de quatro para três, deixando o modelo muito similar ao especificado para o cliente web.

Figura 7: Diagrama de classes utilizadas no Servidor.

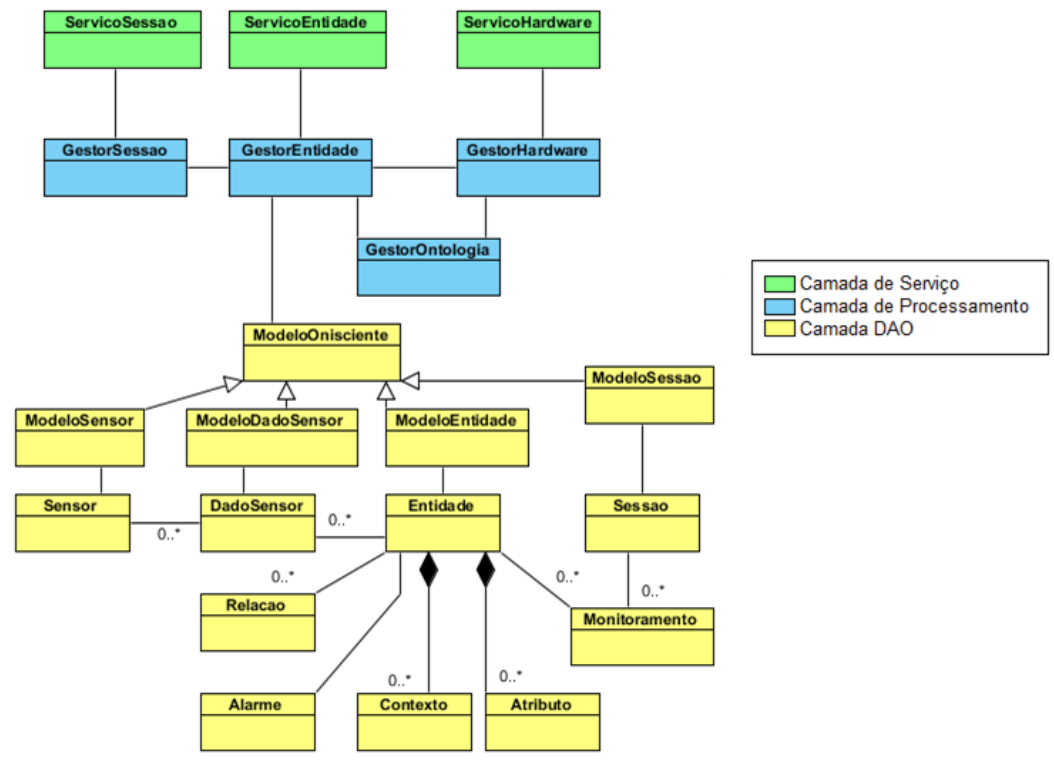

O desenvolvimento do cliente Android resultou na criação de 24 classes, sendo que oito estão relacionadas à camada de interface, representando as telas do sistema, quinze estão na camada de processamento e uma na camada de serviços. Essa divisão pode ser acompanhada por meio da Figura 8, onde a camada de serviços está indicada pela cor vermelha, a camada de interface pela cor verde e a camada de processamento pelas cores amarelo e azul. As classes em azul representam as classes de gestão, enquanto as em amarelo representam as classes de estrutura. Cada classe da camada de interface representa a codificação de uma tela da aplicação. A especificação de cada tela fica armazenada em um arquivo de extensão XML e, para as telas que tiverem um menu, mais um arquivo contendo as suas informações.

Figura 8: Diagrama de classes do cliente Android.

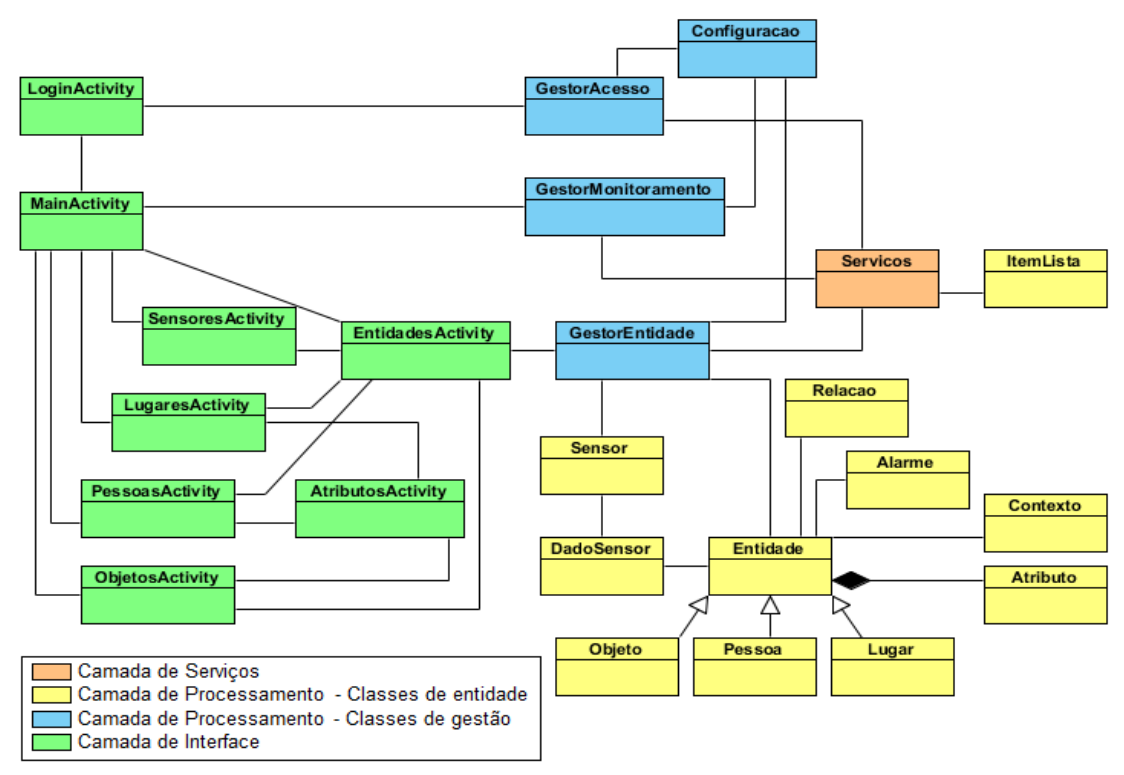


A interface do aplicativo Android foi construída para que o usuário possa ter uma experiência prática, realizando qualquer tarefa desejada em apenas alguns cliques. As telas elaboradas tendem a ser simples, exibindo apenas os componentes necessários para atenderem o objetivo para o qual foram criadas, deixando as opções disponíveis aos usuários por meio de menus. A interface do aplicativo é mostrada na Figura 9.

Figura 9: Exemplos da interface de login, monitoramento, seleção de entidade e seleção de nova entidade do cliente Android.
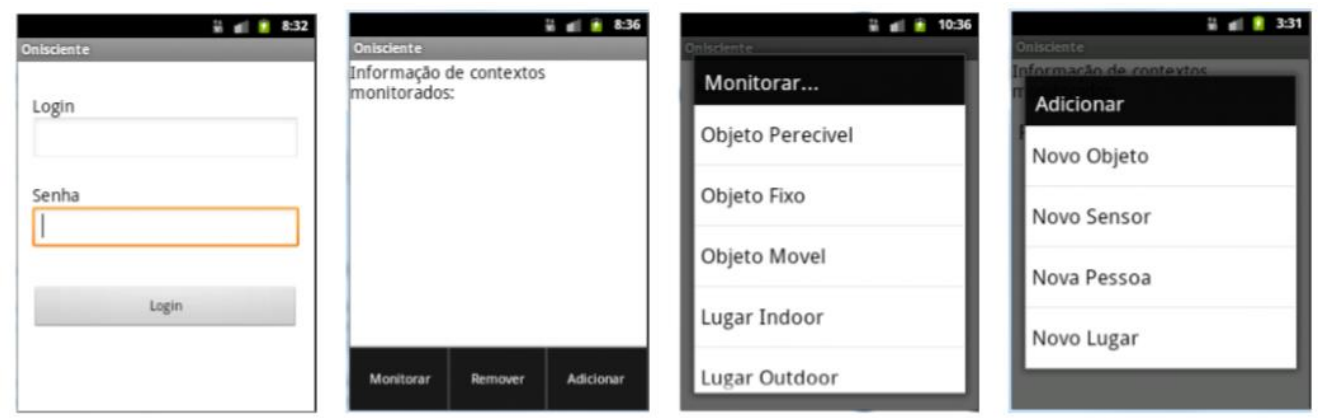

O cliente web é composto por um site web e apresenta-se como um recurso para monitoramento de entidades para indivíduos que não possuam um dispositivo móvel que execute o sistema operacional Android. O cliente web utiliza os mesmos recursos de comunicação que o cliente Android para interação com o servidor do Onisciente. A principal diferença é como as informações e os recursos são apresentados ao usuário, visto que, com mais espaço para apresentação de componentes, o site pode apresentar mais facilidade de uso para o usuário, se comparado ao cliente Android.

A implementação do cliente web seguiu o modelo especificado na seção anterior e gerou um conjunto de classes que pode ser visualizado na Figura 10. Do total de 21 classes, uma está inserida na camada de serviços, sete na camada de interface e treze na camada de processamento. A interface com o cliente web é mostrada na Figura 11, em que os recursos são praticamente os mesmos que o cliente Android pode utilizar.

Figura 10: Diagrama de classes do cliente web.

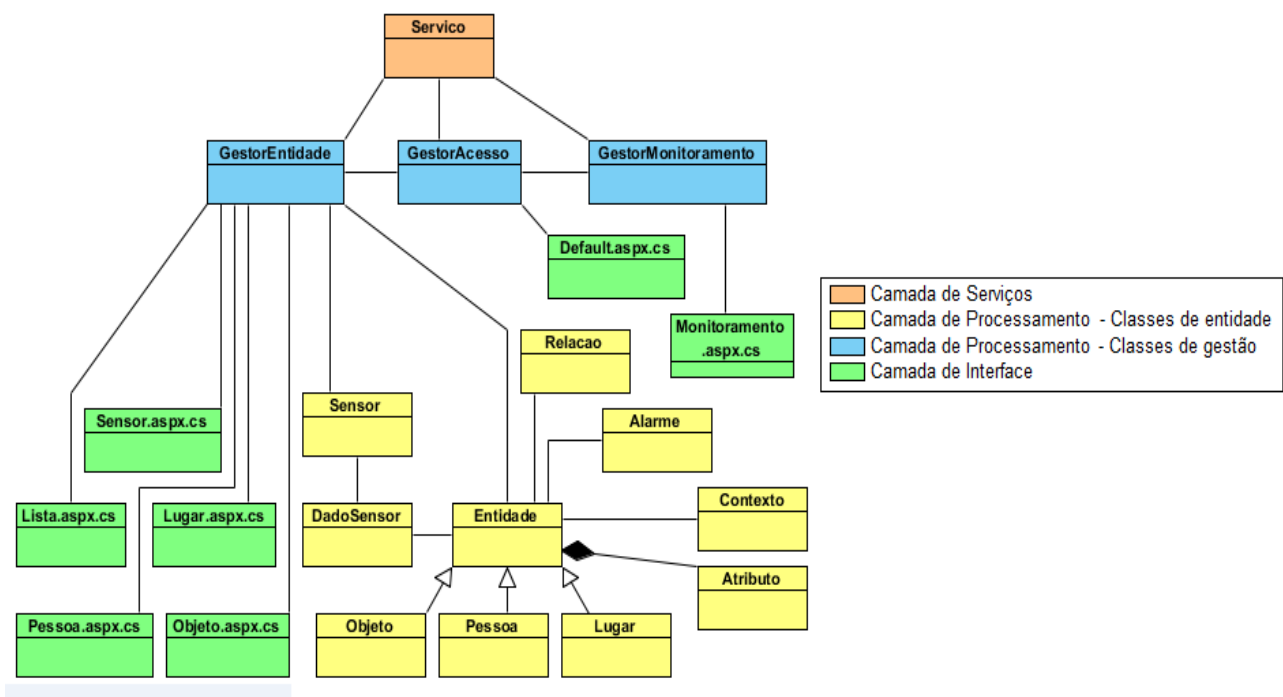


Figura 11: Exemplo da interface do cliente web.
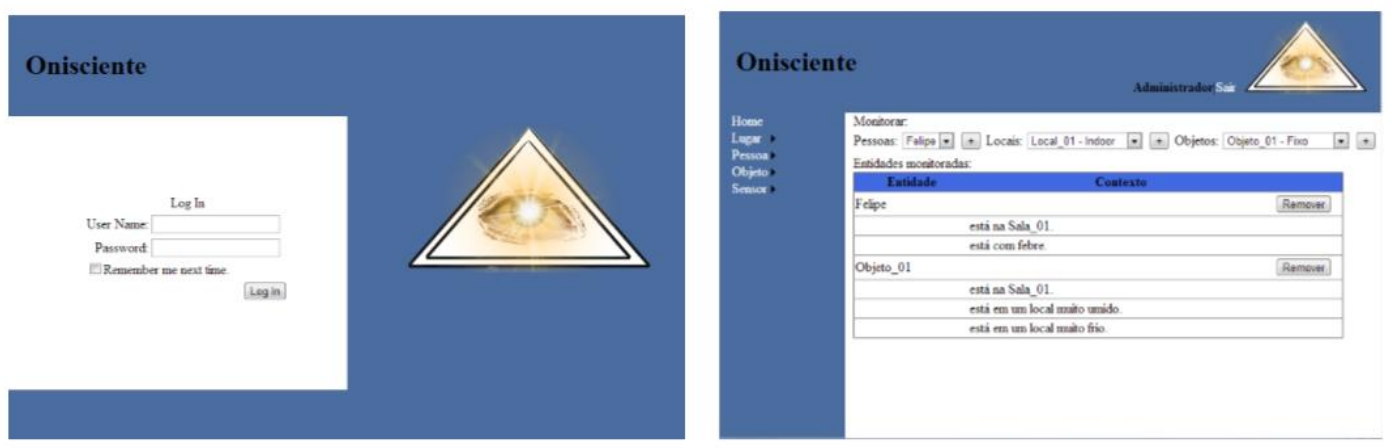

\section{Avaliação por meio de cenários e casos de uso}

A avaliação do Onisciente tem como objetivo, inicialmente, testar a funcionalidade do protótipo usando cenários hipotéticos, além de situações reais de uso. Também tem como finalidade obter métricas para analisar o desempenho do servidor, ao gerar contextos conforme dados são obtidos em pequenos intervalos de tempo. A utilização de cenários tem sido a opção da comunidade acadêmica para avaliar trabalhos em ambientes sensíveis ao contexto e ubíquos $[4,14]$. Os cenários elaborados procuram representar algumas das atividades durante a execução do Onisciente, verificando o comportamento da ontologia, bem como demonstrar a funcionalidade da ontologia durante a aplicação de dados sobre ela. Para isso, foram elaborados três cenários hipotéticos que procuram atender a esse requisito. O processo avaliativo envolve a aplicação de dois casos de uso que representam situações reais que podem ocorrer com entidades.

O primeiro cenário visa demonstrar o funcionamento da ontologia, ao receber informações obtidas pelo acelerômetro, que são aplicadas diretamente sobre uma entidade específica. $\mathrm{O}$ segundo cenário tem como foco avaliar o comportamento da ontologia ao receber informações de temperatura e umidade e qual o efeito que ambas as informações combinadas podem exercer sobre uma pessoa e um objeto. E o terceiro cenário explora a combinação dos dados obtidos com o acelerômetro, temperatura e umidade sobre uma pessoa. O primeiro caso de uso representa uma carga sendo transportada por uma via que varia entre boas e más condições e o segundo caso de uso explora a exposição de entidades às condições climáticas durante um período controlado de tempo.

\subsection{Cenário 1}

"Uma caixa contendo um material considerado frágil, de baixa resistência, que pesa em torno de 1,5Kg é transportada por um caminhão em uma via asfaltada quando este passa por um buraco que sacode a carga arremessando-a para cima a uma força de $2 G$. Ao cair, a caixa rola ficando fora de posição até o término da viagem. Segundo o acelerômetro, os valores dos eixos $x$, y e z registravam respectivamente 0.1,-0.6 e 0.7". Como resultado, a aplicação dos dados descritos no cenário mostrou que o impacto gerado no momento em que a carga foi arremessada acarretou em avaria do seu conteúdo. As informações posteriores ao impacto informaram que a caixa passou o restante da viagem fortemente inclinada, o que caracteriza que a entidade está virada.

\subsection{Cenário 2}

"Em uma construção estão dispostas três salas com temperatura e umidade controladas. Dentro de cada uma dessas salas estão uma pessoa e um objeto, o qual precisa estar em um ambiente com certas condições especificas. A primeira sala é utilizada para resfriar alimentos e mantém uma temperatura de aproximadamente $-5^{\circ} \mathrm{C}$ com umidade de $65 \%$. Ela contém um objeto que suporta temperaturas entre -15 e $2^{\circ} \mathrm{C}$ e umidades variando entre 30 e $80 \%$. A segunda sala está a uma temperatura de $25^{\circ} \mathrm{C}$, com umidade em $30 \%$, e o objeto dentro dela pode ficar a uma temperatura entre 0 e $60^{\circ} \mathrm{C}$ e a uma umidade que varie entre 0 e $50 \%$. A terceira sala contém um forno que, quando aceso, mantém a sala a $40^{\circ} \mathrm{C}$ e a umidade em $15 \%$. O objeto dentro dela suporta temperaturas entre 0 e $100^{\circ} \mathrm{C}$ e umidades entre 10 e $70 \%$. Em um determinado momento, uma queda de 
luz desliga os equipamentos que mantinham a refrigeração do ambiente, causando mudanças na temperatura e umidade à medida que o tempo passa. Após uma hora, a primeira sala alcança uma temperatura de $-1^{\circ} \mathrm{C} e$ umidade de $58 \%$, a segunda $28^{\circ} \mathrm{C}$ de temperatura, mantendo os $30 \%$ de umidade e a terceira sala $51^{\circ} \mathrm{C}$ de temperatura com uma umidade de $13 \%$. Ao final da segunda hora, quando a luz retornou, a primeira sala indicava valores de $4^{\circ} \mathrm{C}$ e $52 \%$ de temperatura e umidade respectivamente, a segunda sala estava com temperatura de $31^{\circ} \mathrm{C}$ e umidade $31 \%$ enquanto a terceira sala alcançou $59^{\circ} \mathrm{C}$ e $9 \%$ de umidade."

$\mathrm{O}$ resultado obtido indicou que apenas na segunda hora, no momento em que a luz retornou, os objetos apresentaram sinais de possíveis avarias. $\mathrm{O}$ objeto da primeira sala acabou sendo exposto a uma temperatura acima do ideal, enquanto o objeto da sala três acabou ficando a uma umidade abaixo do ideal. Os demais objetos não tiveram indicação de que pudessem ter sofrido algum tipo de avaria. Vale destacar que, embora o Onisciente informe que os objetos tenham sido expostos a condições desfavoráveis, não há meios de garantir que eles sofreram algum tipo de dano; o que o sistema informa é uma possibilidade segundo as características da entidade analisada. Quanto às pessoas, o indivíduo que permaneceu na primeira sala esteve com frio durante todo o tempo que se passou; a pessoa que esteve na segunda sala inicialmente estava com frio, mas devido ao aumento de temperatura passou o restante do tempo com sensação de desconforto. Por fim, a pessoa que estava na sala mais quente passou a maior parte do tempo confortável, tendo sensação de desconforto ao final do período.

\subsection{Cenário 3}

"João está andando de moto a uma velocidade de $80 \mathrm{~km} / \mathrm{h}$ em uma estrada asfaltada em ótimas condições durante uma chuva de inverno. No momento, a temperatura está em $13^{\circ} \mathrm{C}$ a uma umidade de $88 \%$."

Ao aplicar os dados na ontologia, foi descoberta que a sensação térmica à qual a pessoa estava exposta era de aproximadamente $1{ }^{\circ} \mathrm{C}$ e, utilizando essa informação para analisar o índice de conforto dessa pessoa, foi possível inferir que, durante o tempo em que esteve na estrada, ela estava passando frio. A ontologia apresenta grande importância para o funcionamento do Onisciente, e os testes realizados até esse momento tiveram como objetivo avaliar a sua capacidade de analisar informações apresentadas e gerar contextos que estivessem em conformidade com as situações especificadas pelos cenários de teste.

\subsection{Caso de uso 1}

O primeiro caso de uso visou explorar a capacidade do Onisciente de determinar se objetos estão sofrendo vibrações e impactos. O teste consistiu em realizar uma rota predeterminada, constituída por trechos bem distintos. O primeiro trecho (Figura 12a cor vermelha) é composto por uma estrada de terra (Figura 12b) de aproximadamente $1,2 \mathrm{~km}$ de extensão, bastante irregular, que possui muita oscilação e buracos. O segundo trecho (Figura 12a cor azul) consiste em uma via asfaltada (Figura 12c) com extensão aproximada de 1,5 km, plana, com algumas lombadas.

Figura 12: Imagens dos trechos por imagem de satélite e imagens reais dos trechos.
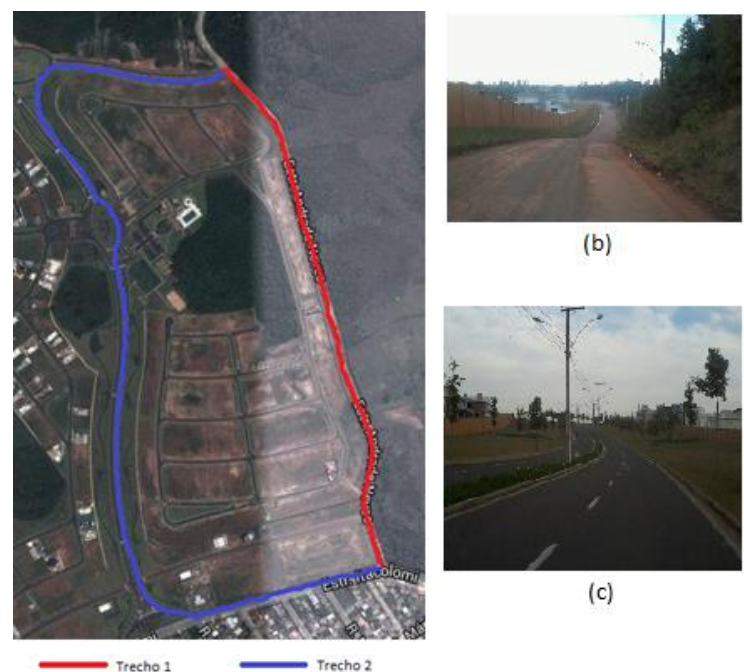

(b)

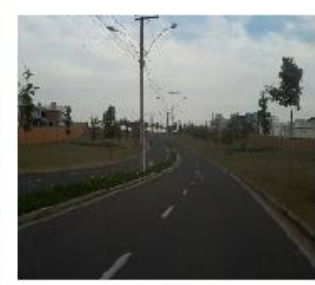

(c)

(a) 
Cada trecho foi percorrido três vezes a uma velocidade média de $40 \mathrm{~km} / \mathrm{h}$. O equipamento de monitoramento foi posto sobre o tampão do porta-malas do veículo utilizado. Para garantir a integridade do equipamento, este foi colocado dentro de uma caixa para protegê-lo de possíveis impactos durante o trajeto. Conforme a realização dos trajetos foi sendo realizada, alguns registros de contextos foram gerados. O primeiro trecho, composto por uma estrada de terra, registrou, nas três passagens, dois registros de impactos, com valores aproximados, com exceção da terceira passagem, que apresentou um valor bem acima dos demais (desvio padrão de 0,32 ). Esses valores podem ser analisados ao verificar a Tabela 3 , onde são informados, para cada passagem do primeiro trecho definido, a quantidade de impactos ocorridos e o valor do maior impacto registrado.

No segundo trecho, composto por uma via asfaltada com algumas lombadas, os resultados obtidos divergiram a cada passada. Na primeira execução do trecho, não foram registradas ocorrências, porém, na segunda passada houve um registro de que a caixa virou e, na terceira, a ocorrência de um impacto. Ambos os registros foram feitos no mesmo ponto do trajeto, em uma pequena lombada para redução de velocidade. A Tabela 4 apresenta os resultados obtidos ao percorrer o segundo trecho do experimento.

Tabela 3: Registro de contextos do trecho 1.

\begin{tabular}{c|c|c}
\hline Passagem & Impactos & Maior Impacto \\
\hline \hline $1^{\mathrm{a}}$ & 2 & 0,64 \\
\hline $2^{\mathrm{a}}$ & 2 & 0,58 \\
\hline $3^{\mathrm{a}}$ & 2 & 1,16 \\
\hline
\end{tabular}

$\mathrm{Na}$ tabela, estão presentes, para cada execução realizada, a quantidade e o tipo de contextos gerados e, se necessário, uma descrição informando um valor relacionado ao contexto. A variação dos resultados obtidos está relacionada à velocidade com que o veículo trafegou pelo trecho no decorrer da execução do trajeto planejado.

Tabela 4: Registro de contextos do trecho 2.

\begin{tabular}{c|c|c|c}
\hline Passagem & Contexto Gerado & Quantidade & Informação \\
\hline \hline $1^{\mathrm{a}}$ & Nenhum & 0 & - \\
\hline $2^{\mathrm{a}}$ & Carga virou & 1 & - \\
\hline $3^{\mathrm{a}}$ & Impacto & 1 & Valor do impacto de 1,09 \\
\hline
\end{tabular}

Durante a realização do caso de uso, notou-se que o desempenho do Onisciente esteve abaixo do esperado para realizar a análise das informações recebidas e processar os contextos. O equipamento utilizado para adquirir as informações foi programado para enviar os dados de maneira seletiva, mas devido à irregularidade do terreno percorrido, havia muitas mudanças nos eixos do acelerômetro, gerando uma grande quantidade de dados que eram enviados ao servidor. O servidor, por sua vez, possui um gargalo no processo de geração de contextos em razão da necessidade de a biblioteca que gera a ontologia recarregá-la antes que se possa realizar as consultas SPARQL. A combinação dessas duas situações acarretou um atraso no processamento que variou de 5 a 10 segundos, conforme o trecho percorrido.

\subsection{Caso de uso 2}

O segundo caso de uso consistiu em um monitoramento das condições climáticas de um ambiente aberto durante um período de tempo em um dia chuvoso de inverno na cidade de Gravataí, localizada no estado do Rio Grande do Sul. Nesse ambiente, era possível encontrar um objeto que apresentava algumas restrições de umidade e temperatura que determinavam os limites aos quais poderia ser exposto. Esse objeto também foi monitorado para se ter conhecimento se as condições às quais ele estava sendo exposto não lhe estavam afetando negativamente.

A aplicação do caso de uso foi realizada durante um período de 14 horas, iniciando-se às $11 \mathrm{~h} 23 \mathrm{~min}$ e encerrando-se à $1 \mathrm{~h} 08 \mathrm{~min}$ do dia seguinte. Nesse intervalo de tempo, foi monitorado um objeto inerte com limites de umidade mínima e máxima de 30 e $60 \%$, respectivamente, o qual deveria estar em uma temperatura que variasse entre 15 e $20^{\circ} \mathrm{C}$. Durante a coleta de dados, a temperatura variou entre $13^{\circ} \mathrm{C}$, no início dos testes, e $17^{\circ} \mathrm{C}$, no final do período. Esses dados podem ser visualizados no gráfico mostrado na Figura 13a. A umidade, por sua vez, obteve variações entre 50 e $62 \%$ e está mostrada no gráfico da Figura 13b. Ambos os gráficos exibem os valores dos dados coletados e indicam o limite inferior e superior de temperatura para o gráfico da Figura 13a, 
bem como o limite superior de umidade do objeto para o gráfico da Figura 13b. Por meio deles, é possível verificar que o objeto esteve exposto a temperaturas abaixo do ideal no início do experimento e que, a partir de um determinado momento, a umidade passou a ser bastante elevada, ficando acima do ideal para o objeto.

Figura 13: Gráfico da variação da temperatura e da variação da umidade.
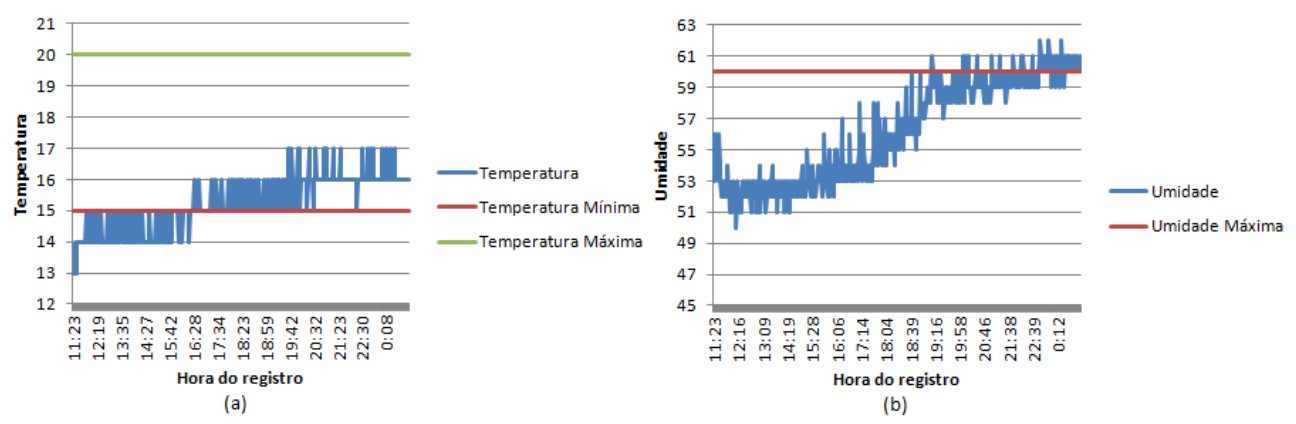

Durante o experimento, foram gerados diversos casos de contexto, totalizando 74 ocorrências de umidade acima do ideal e 225 casos de temperatura abaixo do limite mínimo. Curiosamente, houve quatro ocorrências inválidas de objeto virado durante o período de testes. Essas informações foram resultados de uma falha do acelerômetro ao efetuar a leitura do sensor, já que o objeto monitorado estava completamente inerte e corretamente posicionado sobre uma mesa.

\section{Conclusão}

Este trabalho apresentou o Onisciente, um modelo cujo principal objetivo consiste em gerar contextos a partir de dados variados de diversas fontes, que combinados podem prover informação sobre uma ou mais entidades. No decorrer da evolução deste trabalho, foram apresentadas as especificações do modelo, assim como a descrição de sua implementação e uma avaliação que explorou diversos pontos-chave de sua funcionalidade. Por fim, pôde-se concluir que o Onisciente alcança os objetivos que lhe foram traçados, ao trazer para o usuário final informações que the descrevem a situação do que ele deseja monitorar.

As avaliações revelaram que o Onisciente permite o reconhecimento de situações críticas de entidades monitoradas, quase que em tempo real e de maneira automatizada, não sendo necessária intervenção humana em momento algum do processo de geração de contextos. O processo de geração de contextos foi bastante satisfatório, tendo criado todos aqueles que eram esperados, tanto para os cenários especificados quanto para os casos de uso utilizados e condizentes com a realidade.

Como ponto fraco, o desempenho do processo de geração de contextos ficou abaixo do esperado, levando de 5 a 10 segundos entre a captura dos dados e a geração do contexto. Isso foi ocasionado pelo uso de uma biblioteca de manipulação de ontologias que, quando exigida, não apresentou os recursos suficientes para garantir a eficiência do processo, tendo sido necessário realizar algumas operações de $\mathrm{E} / \mathrm{S}$ no disco rígido do servidor.

Outra oportunidade de trabalho futuro é explorar a total capacidade do modelo, como a possibilidade de utilizar os recursos de localização disponíveis pelas leitoras RFID com um raio de alcance para leitura de tags. Com isso, pode-se incrementar o nível de detalhamento de contextos que tenham como base o posicionamento de entidades.

\section{Agradecimentos}

Os autores agradecem a empresa Sawluz ${ }^{4}$ pelo suporte ao desenvolvimento deste trabalho.

\footnotetext{
${ }^{4}$ Homepage da empresa Sawluz: http://www.sawluz.com.br
} 


\section{Referências}

[1] ROUSSOS, G.; KOSTAKOS, V. RFID in pervasive computing: state-of-the-art and outlook. Pervasive and Mobile Computing. Amsterdam, The Netherlands, v. 5, n. 1, p. 110-131, feb. 2009.

[2] BOUET, M.; SANTOS, A. dos. RFID tags: positioning principles and localization techniques. In: WIRELESS DAYS, 2008. WD ’08. 1ST IFIP, 2008. Anais... [S.1.: s.n.], 2008. p. 1 -5.

[3] NOGUEZ F; COSTA, C. A. BARBOSA. J. L. V. A model integrating virtual worlds and trails. IADIS International Conference Applied Computing, Madrid, Espanha, v. 1, p. 1-8, oct. 2012.

[4] DEY, A. K. Understanding and Using Context. Journal Personal and Ubiquitous Computing. v. 5, 1 (Feb. 2001), 4-7, 2001.

[5] MEHRA, P. Context-Aware Computing: beyond search and location-based services. IEEE Internet Computing, Piscataway, NJ, USA, v. 16, n. 2, p. 12-16, Mar. 2012.

[6] LIU, H.; et al. Survey of Wireless Indoor Positioning Techniques and Systems. Systems, Man ,and Cybernetics, PartC: Applications and Reviews, IEEE Transactions on, Piscataway, NJ, USA, v. 37 , n. 6, p. $1067-1080$, nov. 2007.

[7] OLIVEIRA. Rodrigo R. et al. SWTRACK: An Intelligent Model for Cargo Tracking Based on Off-theShelf Mobile Devices. Expert Systems with Applications, v. 40, p. 2023-2031, 2013.

[8] VIANNA. Henriaue D. : BARBOSA. Jorge L. V. A model for ubiauitous care of non-communicable diseases. IEEE Journal of Biomedical and Health Informatics, v. 18, n. 5, p. 1597-1606, sept. 2014.

[9] MOSTEFAOUI, G. K.; PASQUIER-ROCHA, J.; BREZILLON, P. Context-Aware Computing: a guide for the pervasive computing community. In: THE IEEE/ACS INTERNATIONAL CONFERENCE ON PERVASIVE SERVICES, 2004, Washington, DC, USA. Proceedings... IEEE Computer Society, 2004. p. $39-48$.

[10] SCHILIT, B.; ADAMS, N.; WANT, R. Context-aware computing applications. In: FIRST WORKSHOP ON MOBILE COMPUTING SYSTEMS AND APPLICATIONS, 1994., 1994, Washington, DC, USA. Proceedings... IEEE Computer Society, 1994. p. 85 -90.

[11] HOAREAU, C.; SATOH, I. Modeling and Processing Information for Context-Aware Computing: a survey. New Generation Computing, [S.1.], v. 27, p. 177-196, 2009. http://dx.doi.org/10.1007/s00354-009-0060-5.

[12] WANT, R. An introduction to RFID technology. Pervasive Computing, IEEE, [S.1.], v. 5, n. 1, p. 25 - 33, jan.-march 2006.

[13] SHENG, J.; ZOU, W.; YANG, L.; WANG, B. A RFID-Based Context-Aware Service Model. In: IEEE 10TH INTERNATIONAL CONFERENCE ON TRUST, SECURITY AND PRIVACY IN COMPUTING AND COMMUNICATIONS, 2011., 2011, Washington, DC, USA. Proceedings... IEEE Computer Society, 2011. p. $779-784$

[14] CATARINUCCI, L.; et al. A Context-aware Smart Infrastructure based on RFID Sensor-tags and its Application to the Health-care Domain. In: ETFA, 2009. Anais... IEEE, 2009. p. 1-8.

[15]ZHANG, Y.; ZHENG, Q.; LIU, F. An Extended Context Model in a RFID-Based Context-Aware Service System. In: INTERNATIONAL SYMPOSIUM ON INTELLIGENT INFORMATION TECHNOLOGY APPLICATION WORKSHOPS, 2008., 2008, Washington, DC, USA. Proceedings... IEEE Computer Society, 2008. p. $693-697$.

[16] DELEN, D; SHARDA, R.; HARDGRAVE, B. The promise of RFID-based sensors in the perishables supply chain. Wireless Communications, IEEE, [S.1.], v. 18, n. 2, p. 82 -88, april 2011.

[17] KANG, Y.-S.; et al. A simulation approach for optimal design of RFID sensor tag-based cold chain systems. Journal of Food Engineering, [S.I.], v. 113, n. 1, p. 1 - 10,2012.

[18] GA-ESCRIBANO, J. et al. Improvement in the tracking of special loads by using a three-level RFID system. International Journal of RF Technologies: Research and Applications, [S.1.], v. 3, n. 3, p. 181 199, january 2012.

[19] SATYANARAYANAN, M. Mobile computing: the next decade. SIGMOBILE Mob. Comput. Commun. Rev., New York, NY, USA, v. 15, n. 2, p. 2-10, Aug. 2011.

[20] SAP. Standardized Technical Architecture Modeling. 2007. Disponível em: <http://www.fmcmodeling.org/download/fmc-and-tam/SAP-TAM_Standard.pdf>. Acesso em: 20 abril 2015. 